\title{
Vibration Analysis of Welded Tubular Structures Considering Local Joint Flexibility
}

\author{
Yamin Wang ${ }^{1}$, Yongbo Shao ${ }^{1}$, , Shanshan $\mathrm{Tu}^{2}$, Dongping Yang ${ }^{3}$, Gengqi Niu ${ }^{3}$, Fengle Long $^{3}$ \\ ${ }^{1}$ School of Mechatronic Engineering, Southwest Petroleum University, Chengdu, China \\ ${ }^{2}$ School of Civil Engineering, Yantai University, Yantai, China \\ ${ }^{3}$ Technology Inspection Centerr, China Petroleum \& Chemical Corporation, Dongying, China
}

\section{Email address:}

wangyamin1215@163.com (Yamin Wang),ybshao@swpu.edu.cn (Yongbo Shao), tushanshan912@163.com (Shanshan Tu)

${ }^{*}$ Corresponding author

\section{To cite this article:}

Yamin Wang, Yongbo Shao, Shanshan Tu, Dongping Yang, Gengqi Niu, Fengle Long. Vibration Analysis of Welded Tubular Structures Considering Local Joint Flexibility. American Journal of Mechanics and Applications. Vol. 5, No. 5, 2017, pp. 41-46. doi: 10.11648/j.ajma.20170505.11

Received: November 7, 2017; Accepted: November 23, 2017; Published: December 6, 2017

\begin{abstract}
Welded tubular structure is the backbone of offshore jacket platform. As a thin-walled structure, local joint flexibility (LJF) in a tubular structure is prominent, and it may produce significant effect on the dynamic performance for the overall structure. This study presents a simplified model to analyze the dynamic behavior of a steel tubular structure with LJF. The presented model simplifies a tubular structure into a frame model consisted of beam elements with considering the LJFs at the connections between any two elements. The LJF is simulated with a fictitious beam element (FBE). Methods for defining the dimensions of the cross section and the material properties of the FBE are provided. The accuracy of the presented method is verified through comparing with three dimensional (3D) finite element results on the vibration of a tubular structure. The tested results indicate that LJF has remarkable effect on the vibration of welded tubular structures, and the simplified model presented in this study can provide more accurate estimation compared to conventional rigid frame model.
\end{abstract}

Keywords: Welded Tubular Structure, Local joint Flexibility (LJF), Simplified Model, Fictitious Beam Element (FBE), Vibration

\section{Introduction}

Welded tubular structure is the backbone of an offshore jacket platform. In this structure, circular steel tubes with hollow section are connected through welded joint in which one or several smaller tubes (called brace members) are welded directly onto the surface of a big one (called chord member). As a hollow section tube has much weaker stiffness in its radial direction due to the thin-walled characteristics, the chord deforms easily in its radial direction when a tubular joint is subjected to loading at the brace members. Such deformation is the so-called local joint flexibility (LJF).

LJF is very common in welded tubular structures. However, the welded tubular structure in an offshore jacket platform is generally simplified as a frame structure with rigid connection at the tubular joint for computational effeciency and design brevity, as shown in Figure 1, which indicates that the LJF is not considered in the simplified model. The feasibility of the ignorance of the LJF in a welded tubular structure has to be evaluated to ensure that such simplification has no much influence on the performance of the structure.
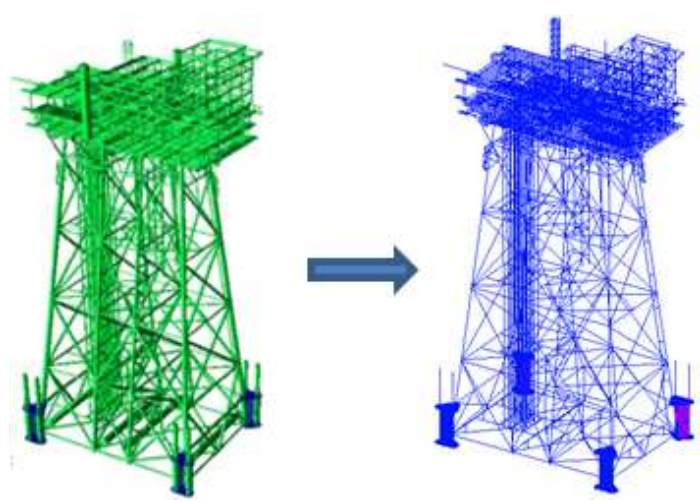

Figure 1. Simplified frame model for an offshore jacket platform. 
In some design guidelines, such as API (2000) [1], it is specified that the LJF has an inevitable effect on the fatigue behavior of a tubular structure, and hence it is necessary to be considered in design. Through experimental investigation, Liu et al. found that the joint rigidity has remarkable effect on the static strength of steel dome structures with welded spherical joints [2]. For welded tubular joints, many studies of the LJF effect on the behavior tubular structures were reported in the literature, and representative ones were presented by many researchers [3-9].

To consider the LJF effect on a tubular structure, the method for calculating of the LJF for different types of tubular joints is necessary to be presented. Many researchers paid their effort in this topic [10-17].

However, previous studies in analyzing the performance of tubular structures considering the LJF have still some problems when they are used in design stage. The most critical problem is the lack of suitable method to apply the presented LJF to the structural analysis of the entire tubular structure. Although some researchers presented revised stiffness matrix of a beam element in the simplified model by introducing the LJF into such stiffness matrix, this method lacks flexibility because the stiffness matrix of each element in the simplified frame model has to be modified when different types of tubular joints are found in the tubular structure.
To overcome such problem, a fictitious beam element is presented in this study to represent the LJF in a tubular structure. With this method, an offshore jacket platform can be simplified into a frame model without losing computational accuracy conveniently. Using the presented model, the vibration of a welded tubular is analyzed. The vibrating process is evaluated through comparison with such process by simulation with three-dimensional (3D) finite element analysis.

\section{Simplified Model of a Welded Tubular Structure}

\subsection{Definition on Local Joint Flexibility (LJF)}

The local joint flexibility can be illustrated in Figure 2, in which a tubular T-joint is subjected to axial compression at the brace. It is clear that a concave deformation on the chord surface around the brace/chord intersection forms. Such local deformation may produce remarkable effect on some performances of a tubular structure, and it is referred to local joint flexibility (LJF) of a tubular joint. Obviously, the LJF becomes larger when the ratio of the chord diameter to the chord thickness, namely a thin-walled tube.

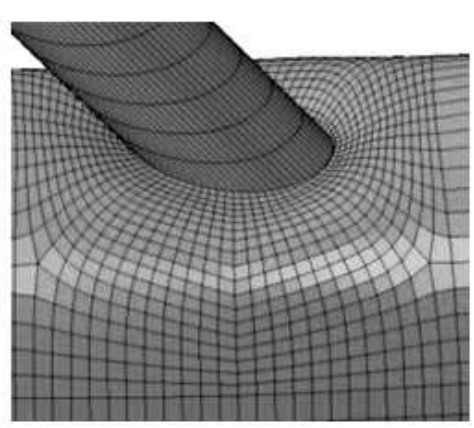

Figure 2. Local deformation at a tubular joint.

To represent the degree of the LJF, definition on the LJF of a tubular joint has to be defined. A tubular joint in a tubular structure is mainly subjected to axial load (tension or compression) and in-plane bending. When the LJFs of a tubular joint under axial load and in-plane bending are denoted with $L J F_{\mathrm{ax}}$ and $L J F_{\mathrm{ipb}}$ respectively, they can are defined and calculated from the following equations:

$$
\begin{gathered}
L J F_{\mathrm{ax}}=\frac{\delta}{P_{\mathrm{ax}}} \\
L J F_{\mathrm{ipb}}=\frac{\phi}{M}
\end{gathered}
$$

where $\delta$ is the axial displacement of the brace at the intersection, $P_{\mathrm{ax}}$ is the axial load at the brace, $\varphi$ is the rotation angle of the brace in the joint plane, and $M$ is the in-plane bending moment at the brace.

As seen in Figure 3, four critical locations, namely a, b, c and $\mathrm{d}$, are marked. Locations a and $\mathrm{c}$ are called crown and locations $\mathrm{b}$ and $\mathrm{d}$ are called saddle. Additionally, three locations, namely e, $\mathrm{f}$ and $\mathrm{g}$, are also marked in Figure 3. They are located at the two sides of the chord, and e, $f$ and $g$ are located on the same cross sections passing through $a, b / d$ and c respectively. Displacements of locations a, b, c, d, e, f and $\mathrm{g}$ in transverse direction of the chord are denoted with $\Delta_{\mathrm{a}}, \Delta_{\mathrm{b}}, \Delta_{\mathrm{c}}, \Delta_{\mathrm{d}}, \Delta_{\mathrm{e}}, \Delta_{\mathrm{f}}$ and $\Delta_{\mathrm{g}}$ respectively, and the intersecting angle between the brace and the chord is denoted with $\theta$. The diameter of the chord is denoted with $D$. $\delta$ and $\varphi$ in Equations (1) and (2) can then be calculated from the follow equations

$$
\begin{gathered}
\delta=\left(\frac{\Delta_{\mathrm{a}}+\Delta_{\mathrm{b}}+\Delta_{\mathrm{c}}+\Delta_{\mathrm{d}}}{4}-\Delta_{\mathrm{f}}\right) / \sin \theta \\
\phi=\frac{\left|\Delta_{\mathrm{a}}-\Delta_{\mathrm{e}}\right|+\left|\Delta_{\mathrm{c}}-\Delta_{\mathrm{g}}\right|}{D / \sin \theta}
\end{gathered}
$$



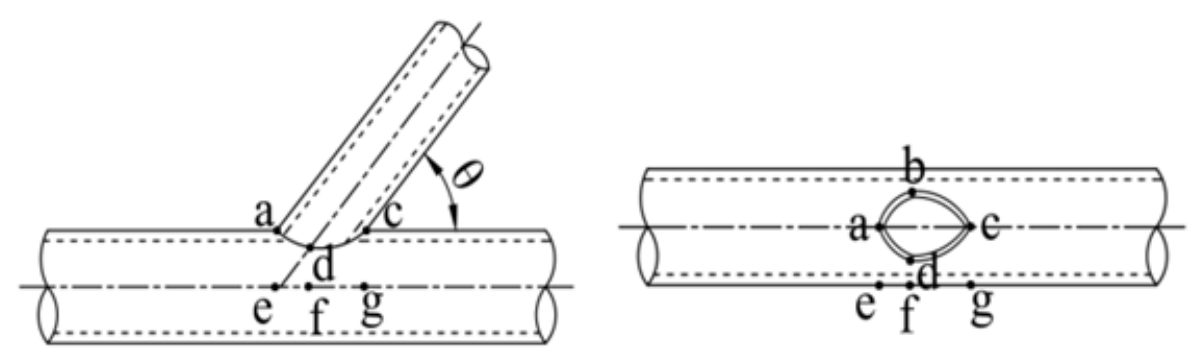

Figure 3. Saddles and crowns in a tubular joint.

\subsection{Simulation of LJF with Fictitious Beam Element (FBE)}

When an offshore jacket platform is simplified into a conventional frame as shown in Figure 1, the tubes are connected together through rigid connection, such as a typical tubular T-joint and a typical tubular Y-joint shown in Figures 4(a) and 4(b) respectively. Such rigid connection ignore the local deformation of the chord under axial load and in-plane bending, and hence the stiffness of the tubular joints are overestimated.

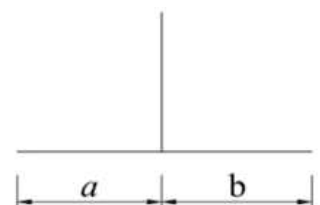

(a) A tubular T-joint

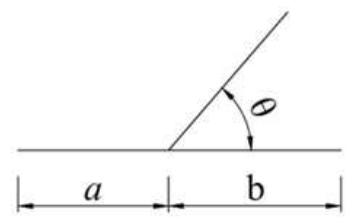

(b) A tubular Y-joint
Figure 4. Rigid connection in a tubular joint

To overcome such problem, a fictitious beam element (FBE) is presented in this study to represent such LJF. After introducing the FBE in a tubular joint, the typical tubular Tand Y-joints are simplified as shown in Figure 5. Compared with conventional rigid connection, a fictitious beam element is added between the brace and the chord. The length in the transverse direction of the chord is equal to the radius of the chord, i.e., $D / 2$. The FBE is used to represent the LJF of the tubular joint, and its material properties and geometrical configuration are necessary to be determined when the simplified model is analyzed.

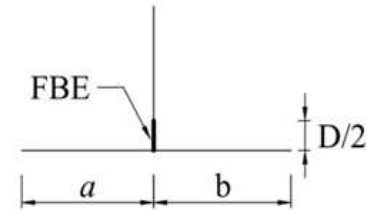

(a) A tubular T-joint

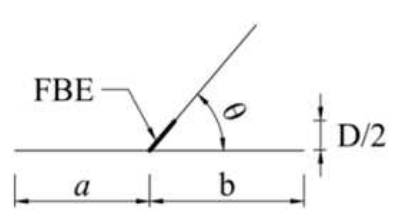

(b) A tubular Y-joint
Figure 5. FBE in a tubular joint.

When a tubular joint is subjected to axial load, the stress and the strain of the FBE are calculated from the following equations

$$
\begin{gathered}
\mathcal{E}=\frac{\delta}{\frac{D}{2} / \sin \theta} \\
\sigma=E \mathcal{\varepsilon}=\frac{P_{\mathrm{ax}}}{A}
\end{gathered}
$$

where $E$ and $A$ are the elastic modulus of the material and the cross section area respectively for the FBE.

From Equations (5)-(6), the cross section area $A$ can be obtained as follow

$$
A=\frac{D}{2 E \cdot \sin \theta \cdot \frac{\delta}{P_{\mathrm{ax}}}}
$$

Substitute Equation (1) into Equation (7), the following equation can be obtained

$$
A=\frac{D}{2 E \cdot \sin \theta \cdot L J F_{\mathrm{ax}}}
$$

Similarly, the following equation is obtained when a tubular joint is subjected to in-plane bending moment

$$
M=\frac{E I \phi}{\frac{D}{2} / \sin \theta}
$$

where $I$ is the moment of inertia for the FBE.

Equation (9) is converted in the following equivalent equation

$$
I=\frac{D}{2 E \cdot \sin \theta \cdot \frac{\phi}{M}}
$$

Substitute Equation (2) into Equation (10), the moment of inertia of the FBE is calculated from the following equation

$$
I=\frac{D}{2 E \cdot \sin \theta \cdot L J F_{\mathrm{ipb}}}
$$

From Equations (8) and (11), the cross section of the FBE can be determined when the elastic modulus of the material $(E)$ and the LJFs of a tubular joint under axial load and in-plane bending moment are provided. The elastic modulus 
of the material can be given with any value. For brevity, such value can be same as that of the chord member. The LJFs of a tubular joint can be calculated from the reported parametric equations in the literature or analyzed directly from a 3D finite element analysis.

After $A$ and $I$ are determined from Equations (8) and (11), the cross section of the FBE can be designed with any shape, i.e., a rectangle or a circle.

\section{Verification on FBE Model}

\subsection{Case Study: A Welded Tubular Structure}

Based on the simulation on the LJF of a tubular joint with FBE method, an offshore jacket platform is easily simplified into a frame structure considering local deformation at the connections. To verify the accuracy of the FBE model in a tubular structure, a typical welded tubular frame as shown in Figure 6(a) is analyzed. This tubular structure is a similar one with that used in practical offshore platforms as shown in Figure 7. The tubular frame as shown in Figure 6(a) is consisted of a series of tubular T-joints. In this study, the left end of the tubular frame is assumed to be fixed and the right end is assumed to be free. A rigid steel plate is connected to the top and to the bottom chords at the right end of the tubular frame. A vertical periodical concentrated force is applied to the top end of the steel rigid plate, and it is expressed as follow

$$
F=60 \sin (4 \pi t)
$$

The units of $F$ and $t$ are $\mathrm{kN}$ and s respectively, and the time lasts for 5 seconds.

The chord and the brace members in Figure 6 (a) are $\phi 219 \times 6$ and $\phi 119 \times 6$ (unit: $\mathrm{mm}$ ) respectively. The density and the elastic modulus of the steels are $7830 \mathrm{~kg} / \mathrm{m}^{3}$ and $210000 \mathrm{~N} / \mathrm{mm}^{2}$ respectively. Other dimensions can be found from Figure 6 (c).

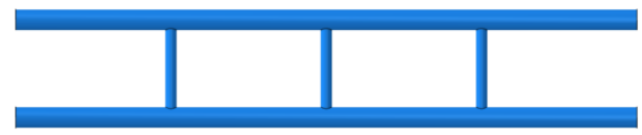

(a) Geometry of a tubular frame

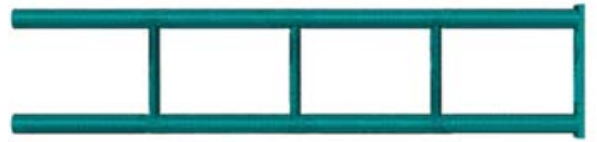

(b) $3 D$ model

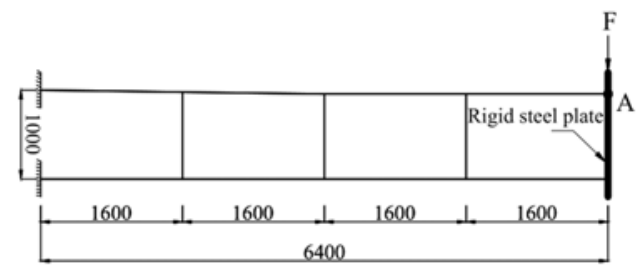

(c) Rigid frame model

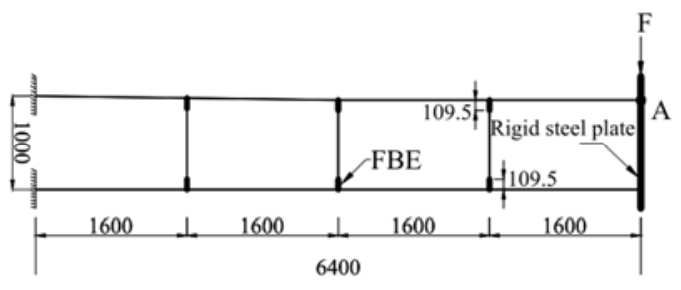

(d) FBE model

Figure 6. Different models of a tubular frame.

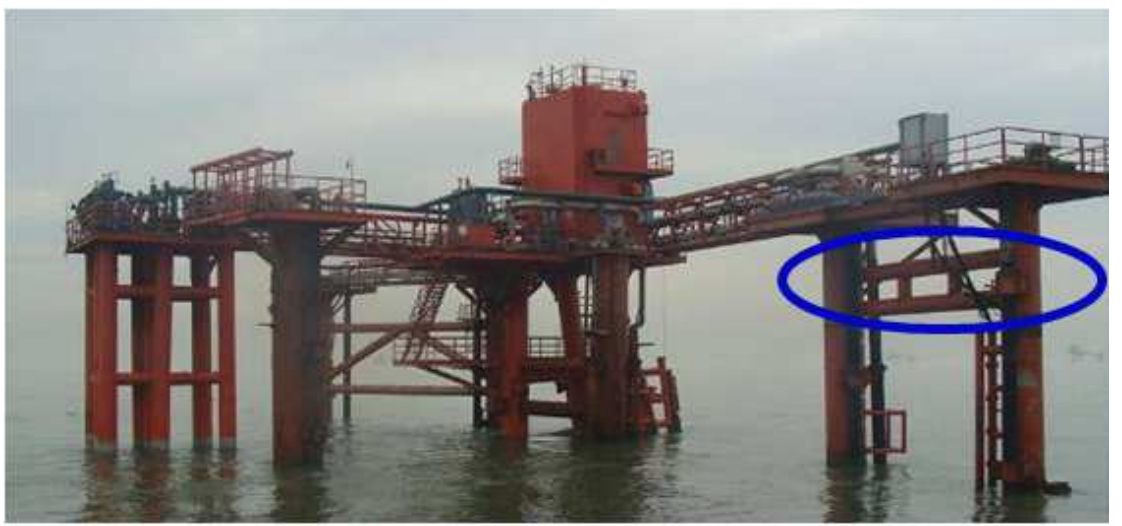

Figure 7. Tubular frame in offshore platforms.

Three types of models are presented to analyze the vibration of the tubular frame under the periodical force in Equation (12). 3D model is the most accurate one because it can simulate every details of the tubular frame, and it is shown in Figure 6 (b). In the 3D model, hexahedron element is used in the discretization of the entire structure, and overall 96296 elements are used in the finite element mesh. The element size far away from the weld is $30 \mathrm{~mm}$ while the element size around the weld is $10 \mathrm{~mm}$ to simulate high stress gradient in this region. The second model is conventional frame model with rigid connection, and beam elements with rigid joints at both ends are used. The overall element number is 1318. In the third model, FBE is used to simulate the LJF, and hence 6 additional FBEs as shown in Figure 6 (d) are used compared to conventional frame model with rigid connection. 
As mentioned previously, definition on the FBE depends on the LJFs, as seen from Equations (8) and (11). In the literature, Fessler et al. presented the LJFs of tubular $\mathrm{T} / \mathrm{Y}$-joints under axial load and in-plane bending moment as follows [18]

$$
\begin{aligned}
& L J F_{A X}^{T, Y}=\frac{1.95 \gamma^{2.15}(1-\beta)^{1.3} \sin ^{1.29} \theta}{E D} \\
& L J F_{I P B}^{T, Y}=\frac{134 \gamma^{1.73} e^{-4.52 \beta} \sin ^{1.22} \theta}{E D^{3}}
\end{aligned}
$$

In Equation (13), $\gamma$ is the ratio of the radius to the thickness of the chord, and $\beta$ is the diameter ratio of the brace to the chord.

For the FBE, its cross section is assumed to be rectangular, and the breadth $X$ and the height $Y$ of the FBE are calculated based on the values of $A$ and $I$ determined from Equations (8) and (11) respectively. The detailed values for some parameters are listed in Table 1.

Table 1. LJFs and geometrical parameters of FBES.

\begin{tabular}{llllll}
\hline $\boldsymbol{L} \boldsymbol{J} \boldsymbol{F}_{(\mathrm{AX})}(\mathbf{m m} / \mathbf{N})$ & $\boldsymbol{L J F}_{(\mathrm{IPB})}(\mathbf{r a d} / \mathbf{N} \cdot \mathbf{m m})$ & $\boldsymbol{A}\left(\mathbf{m m}^{\mathbf{2}}\right)$ & $\boldsymbol{I}\left(\mathbf{m m}^{\mathbf{4}}\right)$ & $\boldsymbol{X}(\mathbf{m m})$ & \\
\hline $7.88 \times 10^{6}$ & $7.93 \times 10^{10}$ & 66.1 & 657539 & 0.191 \\
\hline
\end{tabular}

\subsection{Results and Discussion}

The vibrations of the tubular frame with the above mentioned different models are illustrated in Figure 8 in which the displacement development history with the time is shown. From the comparison in Figure 8, it is found that the frame model with simulation on the LJF by using FBE can produce very similar result compared to the $3 \mathrm{D}$ model. The difference of the displacement amplitudes between the FBE frame model and the $3 \mathrm{D}$ model is only $0.03 \%$. However, the frame model with rigid connection underestimates the displacement amplitude compared to the other two models, which indicates that conventional frame model with rigid connection has higher stiffness and provides unsafe prediction on the dynamic performance of the tubular frame. The difference of the displacement amplitudes between the frame model with rigid connection and the 3D model is as large as $31 \%$. Therefore, the conventional frame model with rigid connection should be used with caution for design purpose.

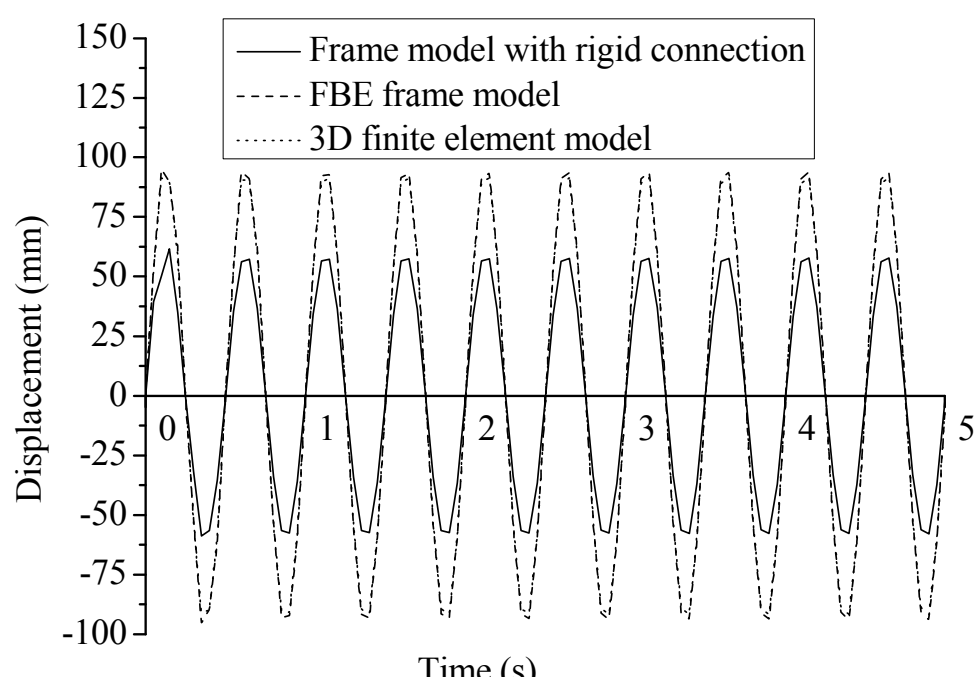

Figure 8. Vibration analyses of the tubular frame with different models.

Using the three different models, the vibration of the tubular frame in 5 seconds is analyzed in a same computer, and thus the computing time can be compared to evaluate the computing efficiency of the three models. The computing periods for the $3 \mathrm{D}$ model, the conventional frame model with rigid connection and the FBE model are 3 minutes, 23 seconds and 25 seconds respectively. It is concluded that the presented FBE model has almost same computing time compared to the conventional frame with rigid connection while it has much shorter computing time compared to the 3D model. Therefore, the presented FBE frame model has the advantages of both high accuracy and computing efficiency.

\section{Conclusion}

Through introducing a fictitious beam element (FBE), the local joint flexibility (LJF) of a welded tubular joint in tubular structures is simulated. The definition and the calculation on the presented FBE are given based on theoretical analysis. Based on the simulation on the LJF of a tubular joint with FBE, a welded tubular structure can be simplified into a frame model consisted of beam elements. Using the presented method, the vibration of a tubular frame is analyzed, and its result is compared with those calculated 
from 3D finite element model and conventional frame model with rigid connection at the joint. The comparison shows that the presented model is reliable and accurate in analyzing the vibration of welded tubular joints while the conventional frame model with rigid connection overestimates the structural stiffness and produces an unsafe prediction.

\section{References}

[1] API. Recommended practice for planning, designing and constructing heliports for fixed offshore platforms. API PR2A-WSD, American Petroleum Institute, USA, 2000.

[2] H. Liu, Z. Chen, S. Xu, Y. Bu, "Structural behavior of aluminum reticulated shell structures considering semi-rigid and skin effect", Steel and Composite Structures, 2015, vol. 54, pp. 121-133.

[3] B. Z. Chen, Y. R. Hu, M. J. Tan, "Local joint flexibility of tubular joints of offshore, structures", Marine Structures, 1990, vol. 3, pp. 177-197.

[4] L. X. Yang, T. Y. Chen, S. Y. Wu, "Local flexibility behavior of tubular joints and its effect on global analysis of tubular structures", China Ocean Engineering, 1990, vol. 4, pp. 371-384.

[5] Y. R. Hu, B. Z. Chen, M. J. Tan, "An equivalent element representing local flexibility of tubular joints in structural analysis of offshore platforms", Computers and Structures, 1993, vol. 47, pp. 957-969.

[6] T. Chen, H. Zhang, "Stress analysis of spatial frames with consideration of local flexibility of multiplanar tubular joint", Engineering Structures, 1996, vol. 18, pp. 465-471.

[7] W. Wang, Y. Y. Chen, "Modeling and classification of tubular joint rigidity and its effect on the global response of CHS lattice girders", Structural Engineering and Mechanics, vol. 21, pp. 677-698.

[8] M. Mirtaheri, H. Ali Zakeri, P. Alanjari, M. Amin Assareh, "Effect of joint flexibility on overall behavior of jacket type offshore platforms", American Journal of Engineering and Applied Sciences, 2009, vol. 2, pp. 25-30.
[9] P. Alanjari, B. Asgarian, M. Kia, "Nonlinear joint flexibility element for the modelling of jacket-type offshore platforms", Applied Ocean Research, 2011, vol. 33, pp. 147-157.

[10] J. G. Bouwkamp, "An improved joint model and equations for flexibility of tubular joints", Journal of Petroleum Technology, 1996, vol. 18, pp. 1491-1499.

[11] Y. Ueda, S. M. H. Rashed, K. Nakacho, "An improved joint model and equations for flexibility of tubular joints", Journal of Offshore Mechanics and Arctic Engineering, 1990, vol. 112, pp. 157-168.

[12] G. Z. Qiu, J. C. Zhao, "Analysis and calculation of axial stiffness of tubular X-joints under compression on braces", Journal of Shanghai Jiaotong University (Science Edition), 2009, vol. 14, pp. 410-417.

[13] G. Z. Qiu, J. H. Gong, J. C. Zhao, "Parametric formulae for axial stiffness of CHS X-joints subjected to brace axial tension", Journal of Zhejiang University - Science A (Applied Physics \& Engineering), 2011, vol. 12, pp. 121-130.

[14] F. Gao, B. Hu, H. P. Zhu, "Parametric equations to predict LJF of completely overlapped tubular joints under lap brace axial loading", Journal of Constructional Steel Research, 2013, vol. 89, pp. 284-292.

[15] F. Gao, B. Hu, H. P. Zhu, "Local joint flexibility of completely overlapped tubular joints under in-plane bending", Journal of Constructional Steel Research, 2014, vol. 99, pp. 1-9.

[16] L. J. Jia, Y. Y. Chen, "Evaluation of elastic in-plane flexural rigidity of unstiffened multiplanar CHS X-joints", International Journal of Steel Structures, 2014, vol. 14, pp. 23-30.

[17] B. Asgarian, V. Mokarram, P. Alanjari, "Local joint flexibility equations for Y-, T and K-type tubular joints", Ocean System Engineering, 2014, vol. 4, pp. 151-167.

[18] H. Fessler, P. B. Mockford, J. J. Webster, "Parametric equations for the flexibility matrix of single brace tubular joint in offshore structures", Proceedings of the Institution of Civil Engineers, 1986, vol. 81, pp. 659-673. 